\title{
Unpacking primary biliary cholangitis and its burden in Japan
}

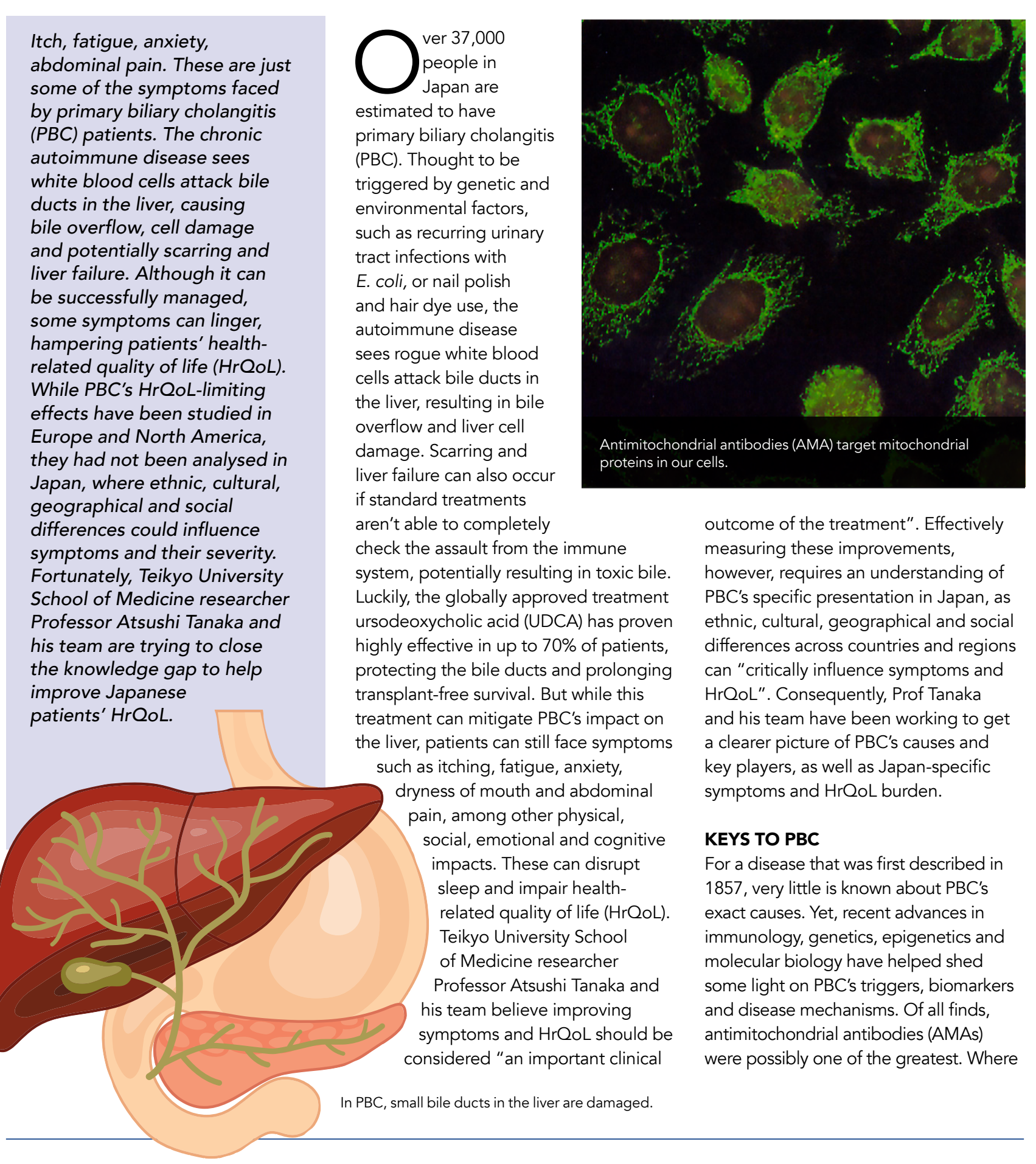

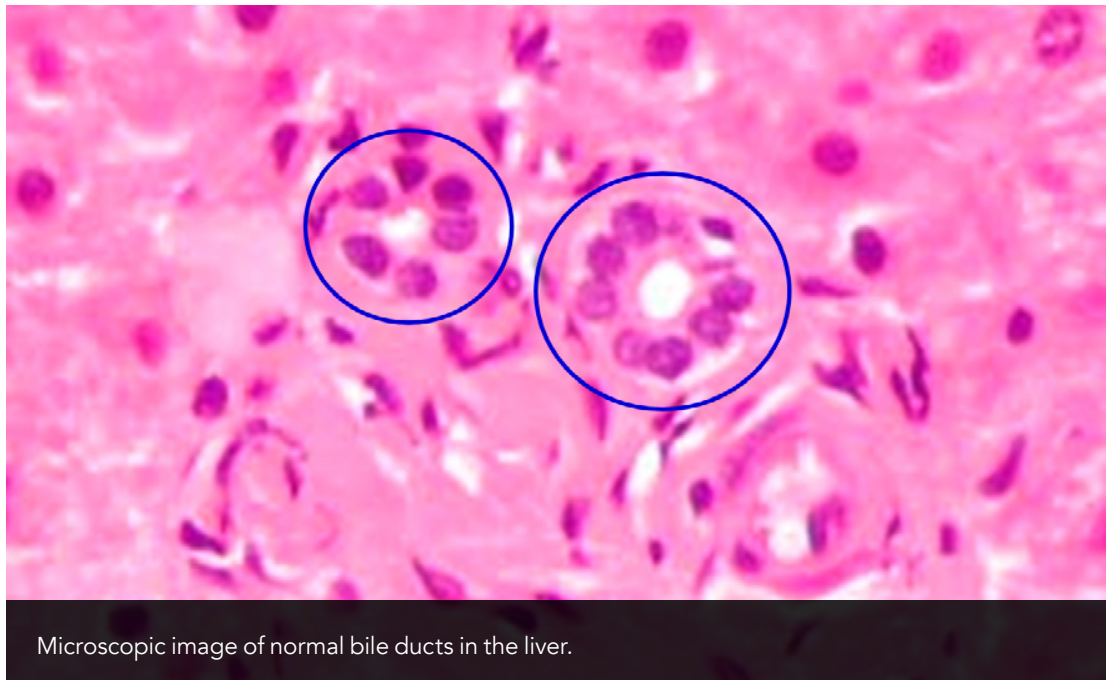

PBC was traditionally diagnosed at later and more severe stages, researchers disease, or potentially discover a patient's risk of developing it, earlier by identifying AMAs in a blood test These humanmade antibodies target mitochondria which perform a specific function within our cells. More specifically, AMAs target one of its proteins and almost exclusively appear
during PBC. It turns out that dying cells in the liver's bile duct lining expose mitochondrial proteins which bind AMAs and spark an Immune response. As white blood cel wage war on these proteins and the proteins are ris and the disease - to escal te.

While genetics and epigenetics play significant roles in PBC development, environmental factors - such as repeated E. coli infections and frequent hair dye, nail polish, other cosmetic and chewing gum use - can help trigger the disease. According to Prof Tanaka and his team, E. coli and these mostly harmless substances contain molecules that can elicit an immune response. They also resemble molecules found in mitochondria. The result: AMAs and

PBC IN JAPAN

Athough PBC presentation and its impacts on HrQoL had been studied in symptoms in and effects on people in Japan. Indeed, background differences can significantly change the way patien experience a disease. This prevents researchers and clinicians from applying optimising patient care. To resolve this, patients across Japan, exploring their symptoms and severity. By translating
PBC's burden in Japan but also compare it to previously studied countries.

According to the researchers, physician considered $88 \%$ of respondents

symptomatic due to a lack of jaundice or abdominal swelling, despite reporting itch, fatigue and cognitive, social and a third of the patients said itching "moderately" to "severely" reduced their HrQoL, while $42 \%$ said the same of fatigue and a quarter expressed high social and cognitive deterioration. Most concerning, however, was the disease's emotional strain, which was felt by over half of the respondents. This was simila to UK studies which showed fatigue to be the biggest burden on patients, con con had yet to define the disease's

Nonetheless, sex and age at diagnosis appeared to be associted with fatigis with females and those diagnosed at a younger age more likely to experience biliary cholangitis. $\quad$ itch. Conversely, itch. Conversely,
the frequently used treatment $\begin{array}{ll}\text { Tanaka and his team surveyed } 496 \text { PBC } & \text { ursodeoxycholic acid (UDCA), did not } \\ \text { patients across Japan, exploring their } & \text { seem to quell the symptoms' severity. }\end{array}$ The results led the researchers to researchers weren't only ablieve PBC symptoms are "primarily

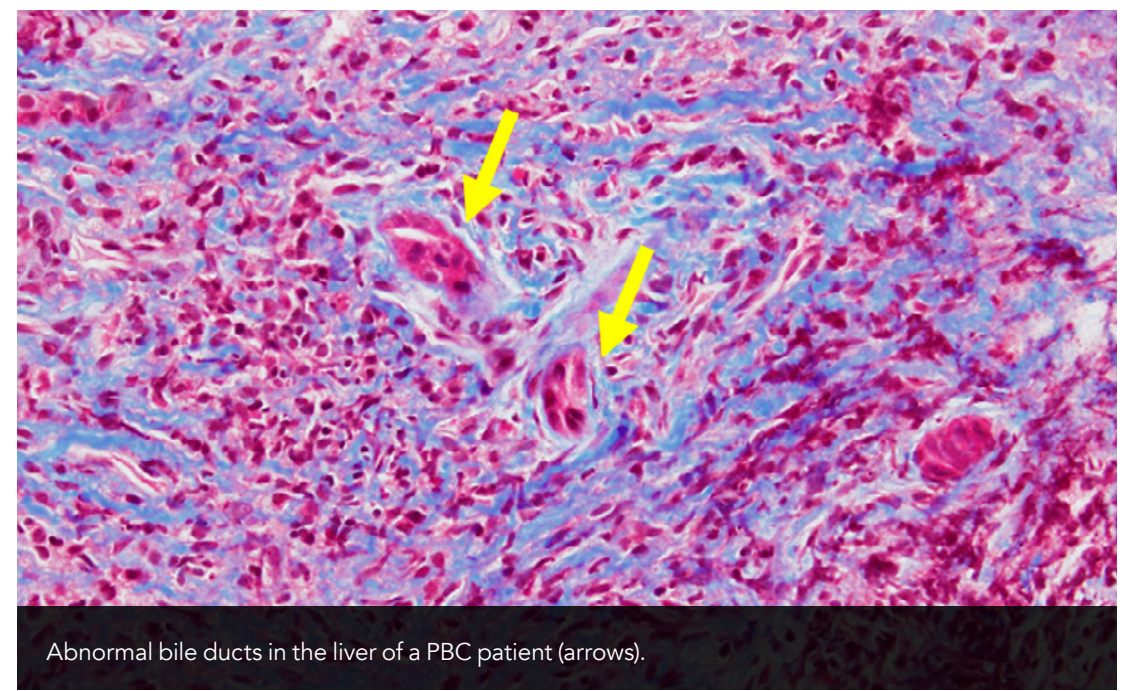




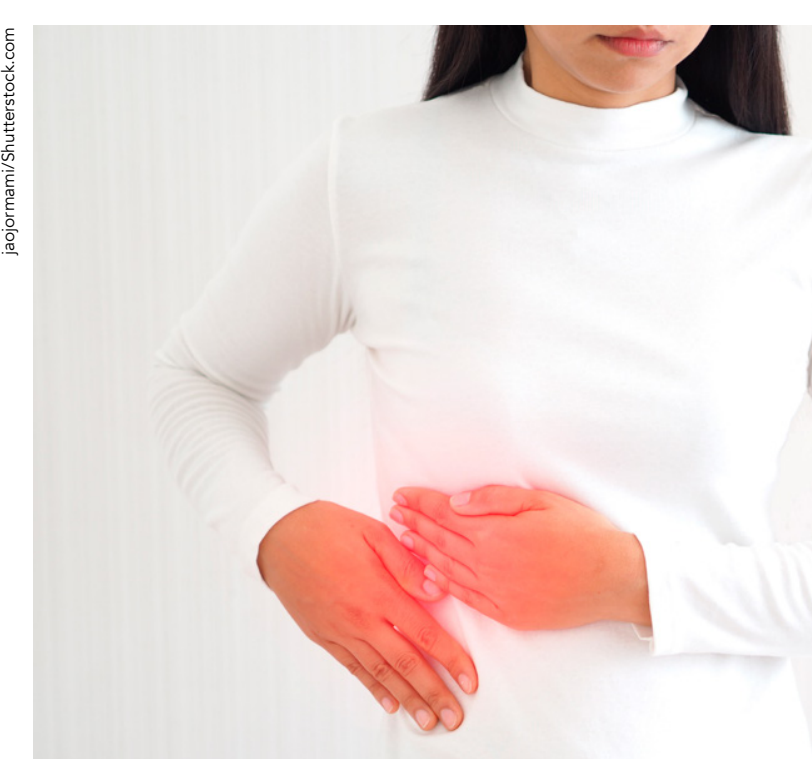

UDCA has proven "completely" effective in treating PBC in most patients,
treatment can cause abdominal fullhess, diarrhoea and constipation.

... physicians considered $88 \%$ of respondents asymptomatic ... despite reporting itch, fatigue and cognitive, social and emotional impairments.

ethnic, cultural and social differences", even though these factors appeared affect the symptoms' strength.

That said and with drugs like UDCA extending life expectancy, Tanaka and his team are urging clinicians to whensively ask patients with PBC whether they experience any symptom ' ways to improve their quality of life.

\section{ALTERNATIVE TREATMENTS}

UDCA has proven "completely"

patients, protecting liver bile ducts and helping patients reach life expectancies comparable to the general population. But it doesn't treat PBC-associated symptoms, and in some cases, this principally safe drug can cause abdominal fullness, diarrhoea and constipation. Further, 30\% of patients show "incomplete responses to the drug, resulting in poor prognoses. Now, to enhance patient outcomes.

To date, fibrates have shown the most promise. Bezafibrate has demonstrated high effectiveness even in UDCA-resistant patients, successfully UDCA-treated pases to "significantly better outcomes" than those without good responses to the drugs. It's even useful for treating PBC induced itch. Moreover combining the drugs has "significantly improved" liver transplant-free survival in PBC patients, particularly those with early-stage disease. Despite its success, Japan has yet to approve bezafibrate for daily clinical use.

Meanwhile, bile-transport inhibitors an nalfurafine have successfully reduced itch in PBC patients, and the latter is already approved for treating the iver diseases.

Although these new drugs have not shown effectiveness in treating other ch could be one of the first steps to boosting HrQoL, and hopefully, more treatments targeting other effects will follow suit.

\section{NOT AS EASY AS ITS NAME} 'PBC' might be easy to say in its . recently, clinicians were unable to diagnose the disease before patients suffered scarring and liver failure. Fortunately, the work of researchers such as Tanaka and his team have helped shed light on the disease, prognoses and a focus shift from treating the illness to its resulting symptoms. Their focus on Japan not only advanced global understanding of PBC but could also support clinicians in raising patients' HrQoL.

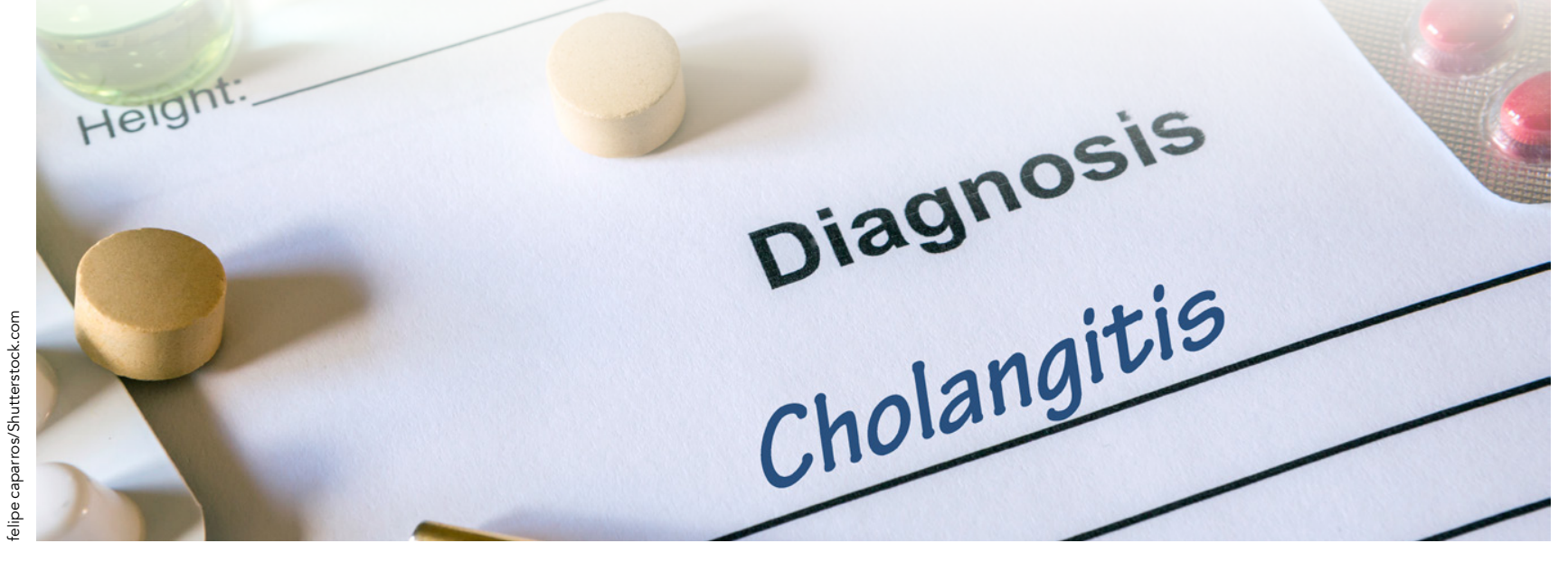

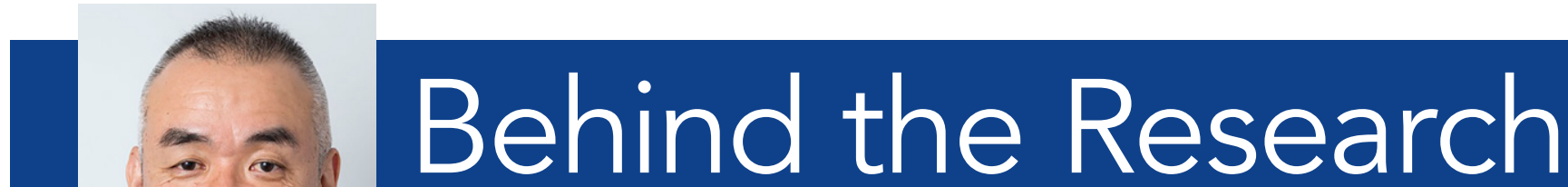

\section{Professor Atsushi Tanaka}

E: a-tanaka@med.teikyo-u.ac.jp T: +81 339641211

\section{References}

- Takahashi, A., Moriya, K., Ohira, H., Arinaga-Hino, T., Zeniya, M., et al. (2018). Health-related quality of life in patients with autoimmune hepatitis: A questionnaire survey. PLOS ONE, Ionline] 13(10). Available at: https://journals.plos.org/plosone

article?id 10

- Tanaka, A. (2019). Emerging novel treatments for autoimmune liver diseases. Heptalogy Research onlinelibrary.wiley.com/doi/abs/10.1111/hepr.13347

- Tanaka, A., Leung, P., Gershwin, M. (2018). Practice \& Research Clinical Gastroenterologt [online] 34-35, 3-9. Available at. https.//wm sciencedirect.com/science/article/abs/pii/ S1521691818300179?via\%3Dihub

- Tanaka, A., Mori, M., Matsumoto, K., Ohira, H. Tazuma, S, et al. (2019). Increase trend in the prevalence and male-to-female ratio of primary biliary cholangitis, autoimmune hepatitis, and primary sclerosing cholangitis in Japan. Hepatology Research, lonline] 49(8). Available at: https:///
onlinelibrary.wiley.com/doi/abs/10.1111/hepr.13342

- Yagi M. Tanka A A Abe, M. Namisaki, T. Yoshij

Yagi, M., Tanaka, A., Abe, M., Namisaki, T., Yosh
H., et al. (2018). Symptoms and health-related quality of life in Japanese patients with primary gitis, Scientific Reports, [online] 8 Avaliable at: https://www.ncbi.nlm.nih.gov/pmc/
articles/PMC6105590/

- Yagi, M., Tanaka, A., Namisaki, T., Takahashi, A. Abe, M., et al. (2018). Is patient-reported outcome with primary biliary cholangitis and refractory pruritus? A post-marketing, single-arm, prospective study. Journal of Gastroenterology, [online] 53(10), 1151-1158. Avallable at. htps:/Mink.springer.com/

- Japan population (live). Ionline] Worldometer. Available at.https.//www.worldometers.info/ world-population

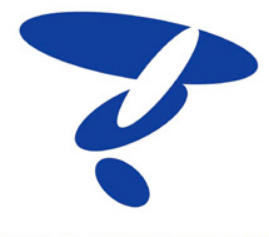

\section{Research Objectives}

Professor Tanaka evaluates health-related quality of life in Japanese patients with primary biliary cholangitis in order to improve this measure of treatment outcome.

\section{Detail}

Atsushi Tanaka

a, Itabashi-ku,

Tokyo, 1738605, Japan

Professor Atsushi Tanaka obtained an MD and PhD degree in 1988 and 1996, respectively, from the University of Tokyo. He stayed at the University of California, Davis from 1996 to Teikyo University School of Medicine.

Funding

The Ministry of Health, Labour, and Welfare of Japan

Collaborators

Tokyo Hepatitis Association

\section{Personal Response}

What do you see as the best-case scenario for or Wh the drection of the treatment? Is there likely pationts multiple drugs?

With life-long treatments such as UDCA and other symptoms to an extent. That being said, we are still far away from our goal to truly cure PBC. The best scenario for future $\mathrm{PBC}$ treatment would be to treat the patient for a limited period of time in order to stop the progression of the illness. Then, symptom origin of PBC. 